\title{
Numerical study of the stress state of a deformation twin in magnesium
}

\author{
M. Arul Kumar ${ }^{1}$, A. K. Kanjarla ${ }^{2}$, S. R. Niezgoda ${ }^{3}$, R. A. Lebensohn ${ }^{1}$, C. N. Tomé ${ }^{1}$ \\ ${ }^{1}$ Materials Science and Technology Division, Los Alamos National Laboratory, Los Alamos, \\ NM 87545, USA \\ ${ }^{2}$ Department of Metallurgical and Materials Engineering, Indian Institute of Technology (IIT) \\ Madras, Chennai, 600036, India \\ ${ }^{3}$ Department of Materials Science and Engineering, The Ohio State University, Columbus, OH \\ 43210, USA
}

\begin{abstract}
We present here a numerical study of the distribution of local stress state associated with deformation twinning in $\mathrm{Mg}$, both inside the twinned domain and in its immediate neighborhood, due to the accommodation of the twinning transformation shear. A full-field elasto-viscoplastic formulation based on Fast Fourier Transformation (FFT) is modified to include the shear transformation strain associated with deformation twinning. We have performed two types of twinning transformation simulations with: (i) the twin completely embedded inside a single crystal, and (ii) the twin front terminating at a grain boundary. We show that: (a) the resulting stress distribution is more strongly determined by the shear transformation than by the intragranular character of the twin or the orientation of the neighboring grain; (b) the resolved shear stress on the twin plane along the twin direction is inhomogeneous along the twin-parent interface; and (c) there are substantial differences in the average values of the shear stress in the twin and in the parent grain that contains the twin. We discuss the effect of these local stresses on twin propagation and growth, and the implications of our findings for the modeling of deformation twinning.
\end{abstract}

Keywords: Deformation twinning, HCP materials, Twinning shear transformation, Crystal plasticity, Local stress distribution.

1. Introduction

1

(C) 2014. This manuscript version is made available under the Elsevier user license http://www.elsevier.com/open-access/userlicense/1.0/ 
Plastic deformation in materials with hexagonal closed packed (hcp) crystal symmetry is usually achieved by a combination of dislocation glide and deformation twinning. Unlike dislocation glide, which reorients the lattice gradually, (10-12) tensile twinning in $\mathrm{Mg}$ is characterized by a localized shear transformation and a nearly $90^{\circ}$ reorientation of the c-axis in a small domain of the crystal. As a consequence of this transformation there is an important stress reaccommodation in the vicinity of the twin, which affects further twin propagation.

Deformation twinning in hcp materials can be rationalized as a three-step sequence: nucleation, propagation and growth (see Fig. 1), and the mechanisms controlling each of these steps are different. Nucleation refers to the process of formation of twin nuclei, and recent molecular dynamics studies suggest that this is a very local phenomenon involving reactions between grain boundary dislocations and stress-driven slip dislocations, combined with atomic shuffling [1-4]. Molecular dynamics results concerning grain boundary nucleation processes were introduced in statistical models, which also include stress fluctuations for deciding which, if any, twin variant is activated in a given grain [5-7].

Propagation refers to the process of the twin front moving into the bulk of the grain and eventually encountering a grain boundary, or an obstacle inside the parent crystal, such as another twin. This paper is focused on elucidating the stress field that develops at the end of the propagation phase when: (i) the twin is completely embedded in its parent grain, or (ii) the twin front is arrested at a grain boundary. In the first case, we study the local stress distribution without any misorientation ahead of the twin front, while in the latter case a significant misorientation may be present in the vicinity of the twin front. In this way, we get insight into how the stress field depends on the misorientation across the grain boundary at which the twin was arrested.

Growth is the process through which the twin interface advances inside the grain and provides significant plastic shear. While growth is driven by the resolved shear stress at the twin/parent interface, such local state is not available when using effective medium homogenization models based on the inclusion formalism. As a consequence, different assumptions are made regarding the stress state that induces twin growth in such homogenization models [8-9]. The present study sheds light on this important aspect of twinning modeling. 
Recent statistical EBSD and modeling studies of deformed $\mathrm{Mg}$ and $\mathrm{Zr}$ [6, 10-12] attribute the frequently reported presence of twin variants with low Schmid factor to a local-stress-controlled nucleation process. In addition, these authors report a correlation between twin thickness and high Schmid factor that they attribute to the fact that better oriented twins can more easily overcome the back-stresses that we explore in this paper. Moreover, small-strain cyclic experiments performed on Mg alloys by Lou et al. [13] and $\mathrm{Wu}$ et al. [14] highlight the role played by de-twinning in accommodating reverse deformation, and suggest that de-twinning is strongly dependent on the local stress states previously induced by twinning.

Experimental measurement of local stress states in polycrystalline materials, which would provide ultimate validation to the simulations reported here, is not a trivial task. Surface-based techniques such as Digital Image Correlations (DIC) measure some components of the local plastic strain tensor on the surface of deforming materials, but they do not provide measurements of the elastic strain components needed for calculating the stress tensor [15]. Attempts at using cross-correlation EBSD technique to measure the elastic strain tensor on the surface of the deformed polycrystalline material proved so far futile due to the extremely high sensitivity of strain error of the technique to the local spin tensor and diffraction geometry, leading to unrealistic values of elastic strains [16-19]. Recent advances in cross-correlation show progress in this area and such measurements may be possible in the future [20].

Also relevant to the present study are recent advances in high-energy X-ray synchrotron experiments, particularly the far field 3D-XRD techniques that allow one to measure in-situ the average elastic strain in a bulk grain. Aydiner et al. [21] employed this technique to monitor the average stress state of a bulk grain and a newly formed deformation twin inside it in AZ31 alloy. Their experiment provides insights into the physics of deformation twinning: upon nucleation, the average value of the resolved shear stress on the twin plane along the twin direction was not only different but of opposite sign in parent and twin. This stress reversal was interpreted to be a consequence of the back-stress induced by internal constraints imposed by the neighboring grains in response to the localized shear associated with the newly formed twin. More recently, Bieler et al. [22] reported similar results for twins in Ti.

In what concerns the incorporation of twinning into crystal plasticity models, it is clear the need 
for knowing the stress at the twin-parent interface, since this stress is the one that drives the twinning dislocations and so twin growth. Macro-scale level viscoplastic and elasto-plastic effective medium models such as the ones used for Mg by Proust et al. [23] and Clausen et al. [8], respectively, rely on the average stress in the parent for activating the twin. The implicit assumption in such procedure is the continuity of the shear stress components parallel to the twin-parent interfaces. While such continuity is physically required at the micro-scale, it does not imply that those particular stress components are constant across the whole grain. As a matter of fact, recent measurements of local elastic strain across parent and twins confirm that elastic strain gradients are present in the vicinity of the twin interface [24]. Moreover, simulations performed by Proust et al. [25] allowed these authors to conclude that predictions of mechanical response upon reload in $\mathrm{Mg}$ were more in line with experiments if the condition of average continuity was relaxed. More recently, Wang et al. [9] found that using the average stress state associated with the inclusion that represents the twin to calculate the resolved shear on the twin interface leads to results in better agreement with $\mathrm{Mg}$ alloys experiments, than when the average stress associated with the parent grain is used.

Crystal plasticity based full field finite element models are capable to predict the local stress state at the twin-parent interface and that can be used for the further twin growth simulation [2627]. But these models are solved under the framework of continuum mechanics at macro scale level to capture the stress-strain response, texture and twin distribution. On the other hand, nano scale level atomistic simulations study the twinning process in the aspect of dislocations interaction with twin-parent interface and/or grain boundary [1-4]. Both macro scale level homogenization models [8-9, 23, 25] and full field models [26-27], and nano scale level atomistic simulations [1-4] fail to capture the local stress state associated with the twinning process at micro scale level, that is experimentally observed by Aydiner et al. [21].

In this paper, we perform local stress calculations at micro scale level in hcp grains using an elasto-viscoplastic formulation based on Fast Fourier Transform (FFT) [28]. We modify the formulation to simulate the transformation strain associated with deformation twinning. We use a continuum approach to solve the equilibrium equation, and no attempt is made here to introduce dislocation theory at the twin-matrix interface [29]. We study the effect of twinning transformation and neighboring grain crystal orientation on local stress distribution. From this 
study we conclude that the twinning transformation plays a more important role on the local stress distribution than the neighboring grain orientation. We also study the back-stress induced in the twin and in the parent grain by the reaction of the medium to the twinning shear transformation. We believe that this back-stress should play a relevant role in controlling twinning/de-twinning during cyclic deformation of hcp materials.

\section{Numerical Method}

The FFT-based formulation used here was originally developed by Moulinec and Suquet [30] as a fast algorithm to compute the local and effective mechanical response of composite materials, in which the source of heterogeneity is related to the spatial distribution of phases with different mechanical properties. The FFT formulation was later adapted by Lebensohn [31] to deal with 3D polycrystals, in which the heterogeneity is related to the spatial distribution of crystals with directional mechanical properties. The formulation has been implemented for polycrystals deforming in different regimes: elasticity [32]; incompressible visco-plasticity [31, 33]; dilatational visco-plasticity [34]; infinitesimal elasto-visco-plasticity [28, 35]; and finite elastovisco-plasticity [36].

The FFT approach consists in iteratively adjusting a compatible strain field, locally related with an equilibrated stress field through a given constitutive relation. The method is based on the fact that the local mechanical response of a heterogeneous medium can be calculated as a convolution integral between the Green function associated with the displacement field of a linear reference homogeneous medium, and a suitably-defined polarization field that contains all information on the heterogeneity and nonlinearity of the given problem. Use is made of Fourier transforms to solve the stress equilibrium equation by reducing convolution integrals in real space to simple products in Fourier space. Thus, for periodic microstructures, the FFT algorithm can be utilized to transform the polarization field into Fourier space and, in turn, to obtain the mechanical fields by transforming back into Cartesian space. However, since the polarization field depends precisely on the a priori unknown strain field, an iterative scheme needs to be implemented. For details on the small-strain elasto-viscoplastic FFT formulation used here, the interested reader is referred to Lebensohn et al. [28].

\subsection{Constitutive Behavior}


In what follows, we consider an elasto-viscoplastic material whose constitutive behavior can be expressed, under an infinitesimal strain approximation, as

$\boldsymbol{\sigma}(\mathbf{x})=\mathbf{C}(\mathbf{x}): \varepsilon^{\mathrm{el}}(\mathbf{x})=\mathbf{C}(\mathbf{x}):\left(\boldsymbol{\varepsilon}(\mathbf{x})-\boldsymbol{\varepsilon}^{\mathrm{pl}}(\mathbf{x})-\boldsymbol{\varepsilon}^{\mathrm{tr}}(\mathbf{x})\right)$

Where $\boldsymbol{\sigma}(\mathbf{x})$ is the Cauchy stress, $\mathbf{C}(\mathbf{x})$ is the elastic stiffness tensor, and $\boldsymbol{\varepsilon}^{\mathrm{el}}(\mathbf{x})$ is the elastic strain at a material point $\mathbf{x}$. The latter is written as the total strain $\boldsymbol{\varepsilon}(\mathbf{x})$ minus the plastic strain $\boldsymbol{\varepsilon}^{\mathrm{pl}}(\mathbf{x})$ due to dislocation slip, minus the transformation strain $\boldsymbol{\varepsilon}^{\mathrm{tr}}(\mathbf{x})$ associated with deformation twinning,. The problem is solved using an implicit time discretization of the form:

$\sigma^{\mathrm{t}+\Delta \mathrm{t}}(\mathbf{x})=\mathbf{C}(\mathbf{x}):\left(\varepsilon^{\mathrm{t}+\Delta \mathrm{t}}(\mathbf{x})-\varepsilon^{\mathrm{pl}, \mathrm{t}}(\mathbf{x})-\dot{\boldsymbol{\varepsilon}}^{\mathrm{pl}, \mathrm{t}+\Delta \mathrm{t}}\left(\mathbf{x}, \boldsymbol{\sigma}^{\mathrm{t}+\Delta \mathrm{t}}\right) \Delta \mathrm{t}-\boldsymbol{\varepsilon}^{\mathrm{tr}, \mathrm{t}}(\mathbf{x})-\Delta \varepsilon^{\mathrm{tr}, \mathrm{t}+\Delta \mathrm{t}}(\mathbf{x})\right)$

Where,

$\dot{\mathcal{E}}^{\mathrm{pl}}(\mathbf{x})=\sum_{\mathrm{s}=1}^{\mathrm{N}} \mathbf{m}^{\mathrm{s}}(\mathbf{x}) \dot{\gamma}(\mathbf{x})$

$\dot{\gamma}^{\mathrm{s}}(\mathbf{x})=\dot{\gamma}_{\mathrm{o}}\left(\frac{\left|\mathbf{m}^{\mathrm{s}}(\mathbf{x}): \sigma(\mathbf{x})\right|}{\tau_{\mathrm{c}}^{\mathrm{s}}}\right)^{\mathrm{n}} \times \operatorname{sgn}\left(\mathbf{m}^{\mathrm{s}}(\mathbf{x}): \sigma(\mathbf{x})\right)$

and:

$\Delta \varepsilon^{\mathrm{tr}}(\mathbf{x})=\mathbf{m}^{\mathrm{tw}}(\mathbf{x}) \Delta \gamma^{\mathrm{tw}}(\mathbf{x})$

during the build-up of the twinning transformation, and 0 otherwise.

In Eqs. (3-5), $\mathbf{m}^{\mathrm{s}}=\frac{1}{2}\left(\mathbf{b}^{\mathrm{s}} \otimes \mathbf{n}^{\mathrm{s}}+\mathbf{n}^{\mathrm{s}} \otimes \mathbf{b}^{\mathrm{s}}\right), \dot{\gamma}$ and $\tau_{\mathrm{c}}^{\mathrm{s}}$ are the Schmid tensor $\left(\mathbf{b}^{\mathrm{s}}\right.$ and $\mathbf{n}^{\mathrm{s}}$ are unit vectors along the Burgers and normal slip directions), the shear rate, and the critical resolved shear stress associated with each of the slip systems 's' respectively, $n$ is the stress exponent, and $\mathbf{m}^{\mathrm{tw}}=\frac{1}{2}\left(\mathbf{b}^{\mathrm{tw}} \otimes \mathbf{n}^{\mathrm{tw}}+\mathbf{n}^{\mathrm{tw}} \otimes \mathbf{b}^{\mathrm{tw}}\right)$ is the Schmid tensor associated with the twinning system ( $\mathbf{b}^{\mathrm{tw}}$ and $\mathbf{n}^{\text {tw }}$ are unit vectors along the twinning direction and the twin plane normal, respectively). The twinning transformation builds-up in increments, until reaching the characteristic twinning 
shear $\mathrm{s}^{\mathrm{tw}}=0.13$ :

$\Delta \gamma^{\mathrm{tw}}(\mathbf{x})=\frac{\mathrm{s}^{\mathrm{tw}}}{\mathrm{N}^{\mathrm{twincr}}}$

for $\mathbf{x}$ in the twin domain and 0 otherwise. The time increment $\Delta \mathrm{t}$ and the number of increments to achieve the twinning transformation $\mathrm{N}^{\text {twincr }}$ are set sufficiently low and high, respectively, to ensure convergence.

\subsection{Simulation details}

In order to study the local distribution of stress associated with twinning transformation first we consider an idealized microstructure, consisting of a tri-crystal where the parent grain (grain 1) is bounded by two neighbors of the same orientation (grain 2) on each side (see Fig. 2). To isolate the effect of twinning transformation on local stress, and uncouple it from the effect of neighborhood crystal orientation, we first consider a Case 0 in which the orientation of the region ahead the twin front is the same as the parent grain (Fig. 2a). This is equivalent to having a twin completely embedded in a single crystal. Next, to study the effect of neighborhood crystal orientation, we assign different orientations to the neighboring grains (Fig. 2b). The orientation of the seven neighbor grains considered here is given in Table I, and labeled Case 0 to Case 6. This tri-crystal is embedded in a homogenous buffer layer where each voxel is assigned a random crystallographic orientation. The $\mathrm{Mg}$ single crystal elastic constants used for the calculations are, in GPa units: $C_{11}=59.75, C_{12}=23.24, C_{13}=21.7, C_{33}=61.7$ and $C_{44}=16.39$. Since the simulation deals with small strains, no work hardening or slip-induced lattice reorientation is considered, except for the explicit reorientation of the twin domain. Easy basal $\{0001\}(11 \overline{2} 0)\left(\tau_{\mathrm{c}}^{\mathrm{s}}\right.$ $=3.3 \mathrm{MPa})$, hard prismatic $\{10 \overline{1} 0\}(11 \overline{2} 0)\left(\tau_{\mathrm{c}}^{\mathrm{s}}=35.7 \mathrm{MPa}\right)$ and hard pyramidal $\{\overline{1} \overline{1} 22\}(\overline{1} \overline{1} 23)($ $\tau_{\mathrm{c}}^{\mathrm{s}}=35.7 \mathrm{MPa}$ ) slip systems are assumed to accommodate plasticity (Beyerlein et al [12]). The simulations involve small macroscopic average strains of order 0.001 , and imposed compressive stresses of up to $60 \mathrm{MPa}$. These values are consistent with the experimental ones of Aydiner et al [21], and local strain or stress values in the vicinity of the twin can be much larger.

The periodic unit cell is discretized into $1225 \times 1225 \times 3$ voxels with a buffer layer 40 voxels thick. 
The discretization scheme is chosen in such a way that the number of voxels is good enough to predict the local micro-field correctly. Further refinement or coarsening of the discretization scheme within a reasonable interval does not change the model prediction significantly. The twin region is chosen arbitrarily with the following considerations. The twin domain is defined by two parallel interfaces chosen to correspond to that of the tensile twin variant (01-12)[0-111]. Different twin thicknesses of 13 and 7 voxels, corresponding to $1 \%$ and $0.5 \%$ of the parent grain volume, were considered. The twin plane is at a $43.1^{\circ}$ inclination with respect to the compression axis and, with an Schmid factor of nearly 0.5 it is favorably oriented for activation. The simulation of the transformation process associated with the twin is carried out in three steps as follows:

1. Initial in-plane compression: The unit cell is subjected to a compression strain of 0.001 $\left(\sigma_{22} \sim-40 \mathrm{MPa}\right)$ achieved in ten incremental steps along direction 2 . This corresponds to mixed boundary conditions of imposed average strain along direction 2 and zero average stress along the other two directions.

2. Twin reorientation: After a compression of $\sim 40 \mathrm{MPa}$, the twinning process is started in the preselected twin voxels. The choice of $40 \mathrm{MPa}$ is arbitrary but suggested by experimental evidence [21]. The first step in the twinning process is the twin reorientation of the preselected set of twin-domain voxels in parent grain.

3. Twinning shear transformation: After the twin reorientation, the local twin shear transformation is enforced to the set of twin-domain voxels that are reoriented in step 2. In order to guarantee convergence, the total shear strain associated with the twinning transformation $\left(\mathrm{s}^{\mathrm{tw}}=0.13\right)$ is imposed using a total of 2000 shear increments. This is a numerical choice made to guarantee convergence and it has negligible rate-dependency effect with twinning shear transformation process. During this process local plastic accommodation takes place in the vicinity of the transforming domain.

4. Further compression up-to $60 \mathrm{MPa}$ : Once the total shear strain in the twin domain is achieved, the simulation cell is subjected to further external straining up-to $\sim 60 \mathrm{MPa}$, which is accommodated by slip.

3. Results 
The EVP-FFT formulation, modified to account for twinning transformation, is applied to predict the local behavior of the tri-crystal unit cells described above. For the fully embedded twin of Case 0 , we assign parent and neighbors the same Euler angles of $\left\{0^{\circ}, 0^{\circ}, 0^{\circ}\right\}\left(\left\{\varphi_{1}, \Phi, \varphi_{2}\right\}\right.$ given in Bunge convention) (see Fig. 2a). Otherwise, we consider six different crystallographic orientations for the neighboring grains while keeping the $\left\{0^{\circ}, 0^{\circ}, 0^{\circ}\right\}$ orientation of the parent grain, and denote these cases as "Case 1" to "Case 6" (see Fig. 2b). The six different orientations are given in Table I and schematically shown in Fig. 2b. The volume fraction of the twin was set to $1 \%$ of the parent grain, corresponding to a twin domain having 13 voxels across its thickness and 409 voxels along its length.

\subsection{Twin embedded inside a single crystal}

First, we present the results of Case 0, i.e., twin completely embedded inside a single crystal. In this case, there is no effect of neighboring grain crystallography on local stress distribution. At the end of the transformation process, the local fields in the tri-crystal system are heterogeneous, with regions of strong localization. As explained earlier, the quantity of most interest is the resolved shear stress on the twin plane along the twin direction, henceforth called the twin resolved shear stress (T-RSS). The contour plots of T-RSS, following the twinning transformation, are shown in Fig. 3 for Case 0. As expected, the T-RSS field is nearly constant across the twin thickness. The strong variations observed in the vicinity of the twin tips are attributable to the reaction of the surrounding neighborhood and its plastic compliance to accommodate the twin shear, and to the non-ellipsoidal shape of the twin. A more detailed picture emerges when one looks at the distributions in the twin domain (Fig. 3b), the neighboring grain (Fig. 3c) and the parent grain (Fig. 3d) separately. Figure 3b clearly shows that the T-RSS is negative inside the twin domain, and fairly homogeneous except at the twin tips. The latter inhomogeneity can be attributed partly to the parallelepiped shape of the twin (since for an elastic ellipsoidal twin it is homogeneous), and partly to the plastic relaxation effect near the twin tip (which affects the purely elastic Eshelby assumption). A sign reversal also takes place in the region of the parent surrounding the twin. Moreover, the profile of the T-RSS along the top interface of the twin, given in Fig. 4, shows large gradients in the vicinity of the twin tip. The shear strain localization at the twin tip is transmitted to the neighboring grain, where it is plastically relaxed by slip. The T-RSS ahead of the tip is lowered to as much as $4 \mathrm{MPa}$, although 
it is not reversed (Fig. 3c).

As mentioned above, Fig. 4 presents the T-RSS profile along the twin-parent top interface in the parent grain and part of neighboring grain, i.e., from point A to point B (shown in Fig. 3a). The T-RSS profile is plotted at: i) the beginning of twinning transformation process under $-40 \mathrm{MPa}$ applied stress; ii) the end of the twinning transformation process; iii) upon further increase in the stress to $-60 \mathrm{MPa}$ without increasing twin volume fraction. It is observed that after reorienting the crystal - but before the shear transformation takes place - the T-RSS is uniform and positive in the twin domain and in the parent. After the twinning transformation is enforced, several important changes take place in the T-RSS profile: a) the profile becomes inhomogeneous both in the parent and in the lateral domains; b) the positive T-RSS in the parent domain becomes negative while the positive T-RSS in the neighbor increases. For twins to grow, and because twinning is directional, the stress state at the twin-parent interface has to be positive. So, the higher T-RSS induced by the twinning transformation process ahead of the twin facilitates twin propagation, but the negative T-RSS induced near the lateral interface tends to suppress twin growth. As a consequence, to grow the twin in the lateral direction one needs further external straining, while the twin can propagate without any further external straining. This may explain the experimental observation that twin propagation proceeds fast until being arrested at a grain boundary, while in order to laterally grow the propagated twin the stress needs to increase. Results being presented later show that the characteristics of this reversal are the same when a neighbor of a different orientation is considered (see later discussion of Fig. 6). Here we only show the T-RSS profiles associated with Case 1 (Fig 4d). Except for minor numerical differences, the profiles are qualitatively the same when the twin is assumed to terminate at a grain boundary.

After simulating the transformation of the $1 \%$ volume fraction twin, the applied stress at the unit cell boundary was raised to $-60 \mathrm{MPa}$ without allowing for further twin growth. As the applied stress increases, the T-RSS at the twin-parent interface shifts from being negative to positive (see Fig. 4c). The T-RSS value at the twin tip is smaller than in the bulk of the twin, but still positive. We speculate that if the twin tends to grow, twinning dislocations emitted at the center of the interface will be arrested at the region of low positive T-RSS values closer to the twin tip/grain 
boundary. As a consequence, this may help explain the characteristic lenticular shape that small twins exhibit close to grain boundaries.

The effect of the twin volume fraction is studied next. While the previous case corresponds to $1 \%$, now we study the results corresponding to a twin of $0.5 \%$ volume fraction of the parent grain. Note that since the shape of the twin is always a parallelogram of fix length, the change in the volume fraction corresponds to a change in the aspect ratio (length/width) of the twin domain. The effect of the twin volume fraction on the average T-RSS in parent and twin is shown in Fig. 5. As the volume fraction of the twin increases, the average values of the T-RSS go from being positive to negative or low positive, both in the parent and the twin. This decrease is faster inside the twin domain. Note that these calculations are done under a fixed applied stress of $-40 \mathrm{MPa}$, and are not meant to represent the actual evolution of a twin as it grows in the grain. In an actual case, the transformed volume and the applied stress should increase simultaneously. We combine the results of the $0.5 \%$ and $1 \%$ volume fraction cases to establish a connection between the present simulation results and the real twin growth situation. Consider the $0.5 \%$ volume fraction case after the twinning transformation, i.e., at $-40 \mathrm{MPa}$ as state $\mathrm{A}$, as represented in Fig. 5. If the material is further loaded from this state A, both stress and twin volume fraction will increase simultaneously. Let us assume that the $1 \%$ volume fraction case is reached at -60 MPa (state B, see Fig. 5). Qualitatively, the evolution of the system from state A to B, shown by an arrow in Fig. 5, can be considered as the T-RSS evolution in a real material with increasing applied stress. An increase in the external compression by -20 MPa increases the average T-RSS in both parent and twin (see Fig. 5), which, as we discuss above, is favorable for activating the motion of twinning dislocations that make the twin grow. The fact that the T-RSS is not uniform along the twin interface and how this may affect the twin growth is discussed later.

At this point, it is interesting to discuss the results of our simulations with the experimental 3DXRD observations of Aydiner et al. [21] who measured the average T-RSS in a grain and in an evolving twin in Mg AZ31. While information about the surrounding grains was not provided by this experiment, the authors reported opposite signs of the average T-RSS in the parent and the twin at a twin volume fraction of $0.6 \%$. With further straining, as the volume fraction of the twin increased, the T-RSS became positive in both the twin and the parent. The predicted effect of the twin volume fraction on the average T-RSS of the twin and the parent is thus consistent with 
these observations. Juan et al. [37] came to a similar conclusion in their recent efforts to model deformation twins using a double inclusion configuration, combined with a Mori-Tanaka scheme. However, since their homogenization approach was purely elastic, their calculated stress values were unrealistically large.

\subsection{Twin front terminating at grain boundary}

While the previous discussion refers to a twin embedded inside a single crystal (Case 0 ) in order to study the effect of twinning transformation alone on the stress distribution associated with twinning, we now study six cases with different neighboring grain orientation to highlight the effect of plastic stiffness and plastic relaxation on neighbor grains and back stresses. The results for all six different cases are qualitatively similar to Case 0 and in Fig. 6 we present the corresponding T-RSS profiles. Complementary numerical results are given in Table I. In all cases we obtain similarly shaped profiles which plateau at T-RSS values ranging from -2.6 to 4.7 MPa (see Fig. 6a). In addition, as the applied stress is increased, the T-RSS at the twin-parent interface shifts from being negative/low positive to positive (see Fig. 6b). The dispersion ranges from 20.8 to $29 \mathrm{MPa}$ and the relative order of the profiles is about the same as was for $-40 \mathrm{MPa}$ compression. Comparison with Case 0 leads to the rather unexpected result that the twinneighbor interaction seems to play a secondary role in shaping the stress field surrounding the twin. Rather, judging from the shape of the profiles for Cases 0 to 6 (Fig 6), the stress variations seem to be more strongly associated with the shear transformation.

If one assumes that twin growth is driven by the resolved shear stress at the twin/parent interface, then two factors will control growth: one is the orientation of the twin with respect to the external stress being applied to the grain (Schmid factor), and the other is the back-stress induced by the transformation. The former will depend on the orientation of the parent with respect to the external applied load, while the latter, shown in Fig. 6, will depend on the neighboring grain crystal orientation. As a consequence, the resolved shear induced by the external applied stress is more likely to overcome the back-stress induced by the twin transformation in grains with high Schmid factor. As a consequence, those twins are more likely to grow and expand. Poorly oriented twins will eventually grow if the macroscopic flow stress increases to a point where the resolved shear can overcome the internal back-stress. Experimental support for this interpretation 
is provided by Beyerlein et al. [11], who report statistical measurements obtained with EBSD showing that thicker twins correlate with higher Schmid factors.

As for the transformation-induced back-stress, it is usually not accounted for in the numerous crystal plasticity simulations of HCP found in the literature, such as the visco-plastic effective medium (mean-field) formulations [6], elasto-viscoplastic effective medium formulations [9, 38], or full-field elasto-viscoplastic formulations [26-27, 39]. Those models treat twinning as a pseudo-slip mechanism, which accommodates shear uniformly across the grain or across the corresponding finite element. The back-stress induced by the localized shear transformation was empirically introduced by Clausen et al. [8] and Saleh et al [40] in their elasto-plastic simulations of Mg AZ31 and a TWIP steel, respectively. These authors show that by doing so their results are consistent with evolution of internal stress in twinned grains measured in-situ by neutron diffraction.

So far we have shown that the resistance of the neighboring grain or region to the localized shear strain associated with the twin formation effectively leads to a back-stress effect on the parent and the twin domains. Such localization can be partially accommodated by slip or twinning in the neighboring grain, via plastic relaxation of the forward stress (i.e. stress ahead of the twin tip). In order to evaluate the effect of local neighborhood crystal orientation upon twin-induced stress configurations we report in Table I several quantitative indicators for the seven cases considered here. These numerical indicators are:

1. The resolved Schmid component along twin direction $\left(\mathrm{m}_{\mathrm{T}-\mathrm{S}}\right)$ of the best oriented slip system in each mode (basal, prism, pyramidal) in the neighbor.

2. The T-RSS (Resolved Shear Stress along the Twin direction) averaged over the twin volume $\left(\tau_{\mathrm{T}-\mathrm{RSS}}^{\mathrm{twin}}\right)$.

3. The T-RSS averaged over the parent volume $\left(\tau_{\mathrm{T}-\mathrm{RSS}}^{\mathrm{parent}}\right)$.

4. The maximum T-RSS along the twin-parent interface along the plateau $\left(\tau_{\mathrm{T}-\mathrm{RSS}}^{\text {plateau }}\right)$ (see Fig 4a).

5. Intuitively, one expects that the back-stress induced on the twin by the resistance to shear localization in the neighboring grains should be proportional to the rigidity of the 
neighbor to plastic relaxation. The latter can be defined by a measure of the forward stress given by the integral $\tau_{\mathrm{FS}}=\int_{\Omega}|\tau| \mathrm{d} \Omega$, where $\tau$ is the T-RSS and $\Omega$ is a domain in the neighboring grain defined by all the voxels that are within a radius of 25 voxels from the center of the twin tip.

6. The average plastic strain resolved along the twin shear direction in the $\Omega$ domain $\left(\mathrm{E}_{\mathrm{T}}^{\Omega}\right)$. This will quantify the plastic accommodation in the neighboring grain along the twin shear direction.

7. The average von Mises equivalent plastic strain in the immediate vicinity of twin-parent grain interface is taken over a 3-voxels thick surrounding the twin interface $\left(\mathrm{E}_{\mathrm{eq}}^{\mathrm{P}-\mathrm{T}}\right)$.

The numerical measures listed above are presented in Table I for all cases. We can see from these results that there is no qualitative difference between Case 0 and the other cases. Again, this confirms twinning shear transformation as the primary cause of the observed local behavior, rather than the neighboring grain orientation. Table I correlates neighboring grain orientation with: a) twin-induced forward stress; b) plastic accommodation in the neighboring grain along the twin shear direction; c) local stress that drives twinning dislocations and so twin growth; d) plastic accommodation in the twin-parent interface.

First we analyze the capability of the deformation modes in the neighbor for accommodating the twinning shear transformation. Third, fourth and fifth columns of Table I give the Schmid component associated with shear on the twinning plane (T-RSS) of the best oriented system of each mode in the neighboring grain. The neighbors in Cases 0, 2 and 3 (group G1) cannot accommodate twin shear with basal slip $\left(\mathrm{m}_{\mathrm{T}-\mathrm{S}}=0\right)$ but they can, in principle, activate prism and pyramidal slip ( $\mathrm{m}_{\mathrm{T}-\mathrm{S}} \sim 0.2$ and 0.4 ). For cases 1, 4, 5 and 6 (group G2) the orientation of the best basal system is on par with the prism and pyramidal ones, with the caveat that the CRSS on basal is much smaller. As a consequence, the accommodation of twinning transformation by the neighbors in group G1 is less favorable compared to G2. This explains the high value of twinning induced forward stress $\left(\tau_{\mathrm{FS}} ; 9^{\text {th }}\right.$ column in Table I) for the cases in $\mathrm{G} 1(0,2,3)$ when compared with the cases in $\mathrm{G} 2(1,4,5,6)$. The same correlation holds for the average plastic strain along the twin shear direction $\left(\mathrm{E}_{\mathrm{T}}^{\Omega}\right)$, which quantifies the plastic accommodation of 
twinning shear transformation by the neighboring grain along the twin shear direction $\left(10^{\text {th }}\right.$ column in Table I).

The back stress on twin and parent induced by the twinning transformation will tend to suppress the twin growth. Although we do not simulate the actual twin growth process here, a qualitative understanding can be gained by correlating the forward stress $\left(\tau_{\mathrm{FS}}\right)$ value with the maximum $\mathrm{T}$ RSS value at the plateau $\left(\tau_{\mathrm{T}-\mathrm{RSS}}^{\text {platea }}\right)$. For the cases G1 with high value of forward stress and less shear accommodation in the neighbor, the T-RSS at the twin parent interface is negative, indicating less relaxation of the transformation strain. Reversely, for the cases in G2 with low value of forward stress, the T-RSS at the twin-parent interface is positive (except for Case 1). A related correlation is provided by the fact that there is systematically more plastic strain in the 3voxel layer adjacent to the twin interface $\left(\mathrm{E}_{\mathrm{eq}}^{\mathrm{P}-\mathrm{T}}\right.$, column 11 in Table I) for G2 (2.4-3.6 x10 $\left.{ }^{-3}\right)$. Since we know that the local stress at the twin-parent interface $\left(\tau_{\mathrm{T}-\mathrm{RSS}}^{\mathrm{plateau}}\right)$ is the one that drives twinning dislocations and so twin growth, we can conclude that growth will be more likely when neighbors belong to $\mathrm{G} 2$ than to $\mathrm{G} 1$ orientations.

There is no clear correlation with the neighbor orientation when the average T-RSS inside the twin $\left(\tau_{\mathrm{T}-\mathrm{RSS}}^{\mathrm{twin}}\right)$ and inside the parent $\left(\tau_{\mathrm{T}-\mathrm{RSS}}^{\text {paren }}\right)$ is compared. In all cases there is a stress reversal within the twin domain following transformation and a large difference between $\tau_{\mathrm{T}-\mathrm{RSS}}^{\mathrm{twi}}$ and $\tau_{\mathrm{T}-\mathrm{RSS}}^{\text {parent }}$, which agrees with experimental observations reported in the literature (Fig. 5c of Aydiner et al. [21]).

Table I. Effect of neighboring grain orientation on twin-induced local behavior: All nine columns (3-12) are defined in the text. The twin volume fraction is $1 \%$.

\begin{tabular}{|c|c|c|c|c|c|c|c|c|c|c|}
\hline Case & $\begin{array}{c}\text { Euler } \\
\text { angles of } \\
\text { neighbor }\end{array}$ & $\begin{array}{c}\mathrm{m}_{\mathrm{T}-\mathrm{S}} \\
\text { for basal } \\
{[\text { Degrees }]}\end{array}$ & $\begin{array}{c}\mathrm{m}_{\mathrm{T}-\mathrm{S}} \\
\text { for prism }\end{array}$ & $\begin{array}{c}\mathrm{m}_{\mathrm{T}-\mathrm{S}} \\
\text { for } \\
\text { pyramida } \\
1\end{array}$ & $\tau_{\mathrm{T}-\mathrm{RSS}}^{\mathrm{twin}}$ & $\tau_{\mathrm{T}-\mathrm{RSS}}^{\mathrm{parnt}}$ & $\tau_{\mathrm{T}-\mathrm{RSS}}^{\text {plateau }}$ & $\tau_{\mathrm{FS}}$ & $\mathrm{E}_{\mathrm{T}}^{\Omega}$ & $\mathrm{E}_{\mathrm{eq}}^{\mathrm{P}-\mathrm{T}}$ \\
\hline 0 & $0,0,0$ & 0.0000 & 0.4330 & 0.2003 & -7.25 & 6.79 & -2.09 & 23.30 & 2.71 & 1.25 \\
{$[\mathrm{MPa}]$} & {$\left[10^{-3}\right]$} & {$[\mathrm{MPa}]$} & \\
\hline
\end{tabular}




\begin{tabular}{|c|c|c|c|c|c|c|c|c|c|c|}
\hline 1 & $75,62,0$ & 0.2658 & 0.2875 & 0.4382 & -8.39 & 5.37 & -3.08 & 14.11 & 3.01 & 2.61 \\
\hline 2 & $30,0,0$ & 0.0000 & 0.4330 & 0.2003 & -5.84 & 6.78 & -2.09 & 23.39 & 2.72 & 1.16 \\
\hline 3 & $0,90,0$ & 0.0000 & 0.2165 & 0.4007 & -6.30 & 6.65 & -2.44 & 22.83 & 2.79 & 1.28 \\
\hline 4 & $0,30,0$ & 0.1875 & 0.3789 & 0.3318 & -0.92 & 7.42 & 4.83 & 12.09 & 3.08 & 3.65 \\
\hline 5 & $45,60,30$ & 0.3750 & 0.2500 & 0.2907 & -4.81 & 4.77 & 0.55 & 12.03 & 4.34 & 2.41 \\
\hline 6 & $50,43,30$ & 0.3125 & 0.3601 & 0.3202 & -3.37 & 7.19 & 2.44 & 13.63 & 3.81 & 3.34 \\
\hline
\end{tabular}

\section{Discussion}

Deformation twinning in hep materials can be thought of as a sequential process of nucleation, propagation and growth, as illustrated schematically in Fig. 1. This paper analyzes the local stress configuration at the end of the propagation stage and correlates the local stresses induced by the localized twin shear transformation with the constraints imposed by parent and neighbor grains. This quest is relevant to the subsequent growth of the twin, since twinning dislocations will be driven by the resolved shear stress acting at the twin/parent interface.

Our calculations show that: a) the T-RSS in the twin is fairly uniform in most of the twin domain, and exhibits steep gradients close to the twin tip due to twin shape and local plastic effects; b) the values of the T-RSS at the parent/twin interface are very different from the average values in the parent and the twin respectively (Table I). These conclusions hold both, when the twin is fully embedded in the parent (Case 0), and when the twin terminates at a neighbor boundary (Cases 1 to 6). While the T-RSS at the interface is the one that drives twin dislocations and makes the twin grow, in effective-medium crystal plasticity models one has only access to the average values in the inclusion domains representing parent or twin. The results of this work suggest that the average twin stress, rather than the average parent stress, better represents the stress at the twin/parent interface. The choice is even more critical when simulating materials subjected to cyclic loading, where twinning and de-twinning are active. Wang et al. [9] show that, if this modeling choice is made, simulations of monotonic, strain path change, and cyclic deformation of $\mathrm{Mg}$ alloys are in much better agreement with the available experimental evidence.

While the current study focuses exclusively on a single twin inside a parent grain, some 
qualitative observations can be made on the possibility of multiple twins and their spacing. After the transformation takes place at $-40 \mathrm{MPa}$, the simulation cell was subjected to a further strain step of 0.001 along direction 2, which requires a final applied stress of -60 MPa. As discussed in section 3, increasing the stress in the unit cell after the twinning transformation increases the shear stress at the twin interface, which goes from being negative to positive (see Fig. 4), which is a necessary requirement for twinning dislocations to propagate and for the twin to grow. In addition, the twin transformation lowers the resolved shear in a domain surrounding the twin (central square in Fig 4b). As a consequence, nucleation and propagation of a newly formed twin from the boundary is unlikely within such critical region surrounding the pre-existing twin, the size of which defines a characteristic twin separation.

It is appropriate to discuss at this point the earlier work by Zhang et al. [41-42] on the local calculation of the stresses at the end of the twin propagation. These authors have performed a phenomenological finite element simulation for understanding the local stress distribution as a consequence of twin nucleation in zirconium. In their simulations, while both the elastic and plastic anisotropy was accounted for, the latter was not achieved by crystallographic slip. Instead, an anisotropic continuum yield function of the Hill type was utilized. Also, since the parent domain was embedded in a homogenous medium there was no influence of local neighborhood. One of the common features presented here and in Zhang et al. work is the profile of the shear stress inside the twin domain (see Fig. 4a), that is, the upside-down parabola with the stress levels dropping drastically close to the twin tips. This could be, as also pointed out in Zhang et al. [41], partly responsible for the lenticular shape commonly observed in twins. Since the shear stress at the twin tips exhibits large negative values, the glide of twinning dislocations is inhibited at the tip, so leading to a lenticular shape when twin growths.

The quasi-3D tri-crystal system solved in this work is a simplified representation of reality but constitutes a necessary first step to identify the features that are relevant to understanding the effect of shear transformation and neighbor presence on twin growth. Future work will rely on a more realistic 3D microstructure of grain and neighbors, and will tackle the nucleation, propagation and growth processes. Specifically, it would be interesting to see how the material responds locally when an initial twin nucleus is created at one grain boundary and allowed to propagate across the grain to the other grain boundary in a sequential process by slowing adding 
the layer of voxels to the propagating nucleus. Work along these lines has been pursued recently by Abdolvand et al. [43-44] but without accounting for the shear transformation effects, which we show are very important. If successful, this simulation may also yield information the process of twin formation at grain boundaries due to an impinged twin. We foresee that the twinning transformation will induced local stress in the neighboring grain in the vicinity of twin tip across the grain boundary, and will help to nucleate new twin variants in the neighbor. Such configurations of adjoining twin pairs have been observed experimentally in hcp Mg and Ti [11, 22 , 39]. From this, we can study the combined effect of twinning transformation and lattice misorientation on twin-twin formation across the grain boundary.

A more direct comparison of these simulation results with experimental data would be feasible using state-of-the-art diffraction characterization techniques. A step along this line is the successful measurement of the local variation of one of the elastic strain components along a line across the bulk of a grain containing twins in a polycrystalline AZ31 sample using Differential Aperture X-ray Microscopy and a monochromatic beam of fixed energy [24]. It would be in principle possible to measure with monochromatic beams of different energies the complete elastic strain tensor and the corresponding stress tensor. Concurrently, efforts are underway to combine the near field High Energy Diffraction Microscopy (nf-HEDM) [45] with the far field 3DXRD technique [46]. While the former measures the spatially resolved orientation fields from the bulk, the latter measures the elastic strains averaged over a given volume in the bulk. These experiments would not only provide us with hitherto unavailable in-situ information on the local micromechanical fields associated with twin nucleation and growth, but would also represent a unique opportunity for the modeling community to rigorously validate their models, not just in terms of their macroscale predictions, but at a much more stringent spatially-resolved local scale.

\section{Summary}

We present here a numerical study of the local distribution of the stresses induced by twinning in hexagonal Magnesium. An FFT-based elasto-viscoplastic formulation was modified to include the transformation strain associated with deformation twinning. The effect of both twinning shear transformation and neighboring grain crystallography on local stress distribution was studied in detail. No attempt is made here to make a dislocation theory analysis of the twinmatrix interface and associated stresses [29]. 
The case of a twin embedded in a single crystal was considered to quantify the effect_of twinning transformation alone on local stress distribution, with free from the effect of neighboring grain orientation on twinning stress. The quantity of interest is the resolved shear stress on the twin plane along the twin direction, which is not homogeneous inside or in the vicinity of the twin. The average values of resolved shear stress in the parent single crystal and in the twin are significantly different. In this regard, the predicted results support the experimentally observed stress reversal upon twinning transformation, during which the positive resolved shear stress in the parent region reverses to negative, while the positive resolved shear stress in the neighboring region increases. From the perspective of the stress acting at the twinmatrix boundary being responsible for twin propagation and growth, our results suggest that the twinning transformation process facilitates twin propagation and hinders twin growth. The resolved shear stress profile along the twin surface provides a reason for experimentally observed lenticular twin shapes.

The simulation using different neighboring grains is performed to study the effect of neighbor misorientation on local stress distribution. The predictions are qualitatively similar to the single crystal case. The present numerical study shows that the twinning transformation plays a primary role on the local behavior, compared with the secondary effects associated with the twin volume fraction or induced by the orientation of neighboring grains.

Acknowledgments

This work was entirely funded by US Department of Energy, Office of Basic Energy Sciences (OBES) FWP-06SCPE401.

\section{References}

[1] Wang J, Beyerlein IJ, Tomé CN. Scripta Mater 2010;63:741.

[2] Wang J, Liu L, Tomé CN, Mao SX, Gong SK. Mater Res Lett 2013;1:81.

[3] Wang J, Yadav SK, Hirth JP, Tomé CN, Beyerlein IJ. Mater Res Lett 2013;1:126.

[4] Wang J, Beyerlein IJ. Model Simul Mater Sc 2012;20:024002. 
[5] Beyerlein IJ, Tomé CN. P R Soc A 2010;466:2517.

[6] Niezgoda SR, Kanjarla AK, Beyerlein IJ, Tomé CN. Int. J. Plasticity 2013;56:119.

[7] Niezgoda SR, Beyerlein IJ, Kanjarla AK, Tomé CN. J Metals 2013;65:419.

[8] Clausen B, Tomé CN, Brown DW, Agnew SR. Acta Mater 2008;56:2456.

[9] Wang H, Wu PD, Tomé CN, Wang J. Mat Sci Eng A 2012;555:93.

[10] Capolungo L, Marshall PE, McCabe RJ, Beyerlein IJ, Tomé CN. Acta Mater 2009;57:6047.

[11] Beyerlein IJ, Capolungo L, Marshall PE, McCabe RJ, Tomé CN. Philos Mag 2010;90:2161.

[12] Beyerlein IJ, McCabe RJ, Tomé CN. J Mech Phys Solids 2011;59:988.

[13] Lou XY, Li M, Boger RK, Agnew SR, Wagoner RH. Int J Plasticity 2007;23:44.

[14] Wu L, Agnew SR, Ren Y, Brown DW, Clausen B, Stoica GM, Wenk HR, Liaw PK. Mat Sci Eng A 2010;527:7057.

[15] Padilla HA, Lambros J, Beaudoin AJ, Robertson IM. Int J Solids Struct 2012;49:18.

[16] Britton TB, Wilkinson AJ. Ultramicroscopy 2011;111:1395.

[17] Britton TB, Wilkinson AJ. Ultramicroscopy 2012;114:82.

[18] Maurice C, Driver JH, Fortunier F. Ultramicroscopy 2012;113:171.

[19] Niezgoda SR, McCabe RJ, Tomé CN. Scripta Mater 2012;67:818.

[20] Wilkinson AJ, Britton TB, Jiang J, Karamched PS. In IOP Conference Series: Materials Science and Engineering 2014;55:012020.

[21] Aydiner CC, Bernier JV, Clausen B, Lienert U, Tomé CN, Brown DW. Phys Rev B 2009;80:024113.

[22] Bieler TR, Wang L, Beaudoin AJ, Kenesel P, Lienert U. Metall Mater Trans A 2014;45:109.

[23] Proust G, Tomé CN, Kaschner GC. Acta Mater 2007;55:2137.

[24] Balogh L, Niezgoda SR, Kanjarla AK, Brown DW, Clausen B, Liu W, Tomé C.N. Acta Mater 2013;61:3612. 
[25] Proust G, Tomé CN, Jain A, Agnew SR. Int J Plasticity 2009;25:861.

[26] Abdolvand H, Daymond MR. J Mech Phys Solids 2013;61:783.

[27] Abdolvand H, Daymond MR. J Mech Phys Solids 2013;61:803.

[28] Lebensohn RA, Kanjarla AK, Eisenlohr P. Int J Plasticity 2012;32:33.

[29] Mitchell T E, Hirth J P. Acta metall mater 1991:39:1711.

[30] Moulinec H, Suquet P. Comput Method Appl M 1998;157:69.

[31] Lebensohn RA. Acta Mater 2001;49:2723.

[32] Brenner, R., Lebensohn, R.A., Castelnau, O. Int J Solids Struct 2009;46:3018.

[33] Lebensohn, RA., Brenner, R, Castelnau, O, Rollett, AD. Acta Mater 2008;56:3914.

[34] Lebensohn RA, Idiart MI, Ponte Castañeda P, Vincent PG. Philos Mag 2011;91:3038.

[35] Kanjarla AK, Lebensohn RA, Balogh L, Tomé CN. Acta Mater 2012;60:3094.

[36] Eisenlohr P, Diehl M, Lebensohn RA, Roters F. Int J Plasticity 2013;46:37.

[37] Juan PA, Berbenni S, Capolungo L. Acta Mater 2012;60:476.

[38] Wang H, Wu PD, Tomé CN, Wang J. Int J Solids Struct 2012;49:2155.

[39] Fernández A, Jérusalem A, Gutiérrez-Urrutia I, Pérez-Prado MT. Acta Mater 2013;61:7679.

[40] Saleh AA, Pereloma EV, Clausen B, Brown DW, Tomé CN, Gazder AA. Acta Mater 2013;61:5247.

[41] Zhang RY, Daymond MR, Holt RA. Mat Sci Eng A 2008:473;139.

[42] Zhang RY, Daymond MR, Holt RA. Mat Sci Eng 2011;528:2725.

[43] Abdolvand H, Daymond MR. Acta Mater 2012;60:2240.

[44] Abdolvand H, Daymond MR, Mareau C. Int J Plasticity 2011;27:1721.

[45] Pokharel R, Lind J, Kanjarla AK, Lebensohn RA, Li SF, Kenesei P, Suter RM, Rollett AD. Annual Review Condens Matter Phys 2014;5:317. 
[46] Lienert U, Li SF, Hefferan CM, Lind J, Suter RM, Bernier JV, Barton NR, Brandes MC, Mills MJ, Miller MP, Jakobsen B, Pantleon W. J Microsc 2011;63:70. 

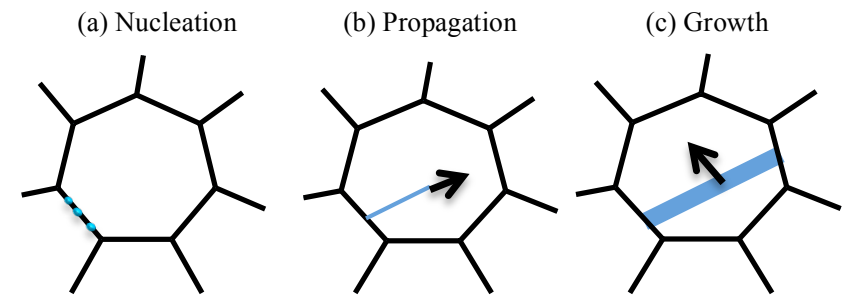

Figure 1: Schematic of the three steps involved in the deformation twinning process: (a) Nucleation: formation of twin nuclei at the grain boundaries. (b) Propagation: transformation of a stable twin nucleus into a lenticular twin propagating towards the other side of the grain; (c) Growth: increase of twin thickness upon further strain. 

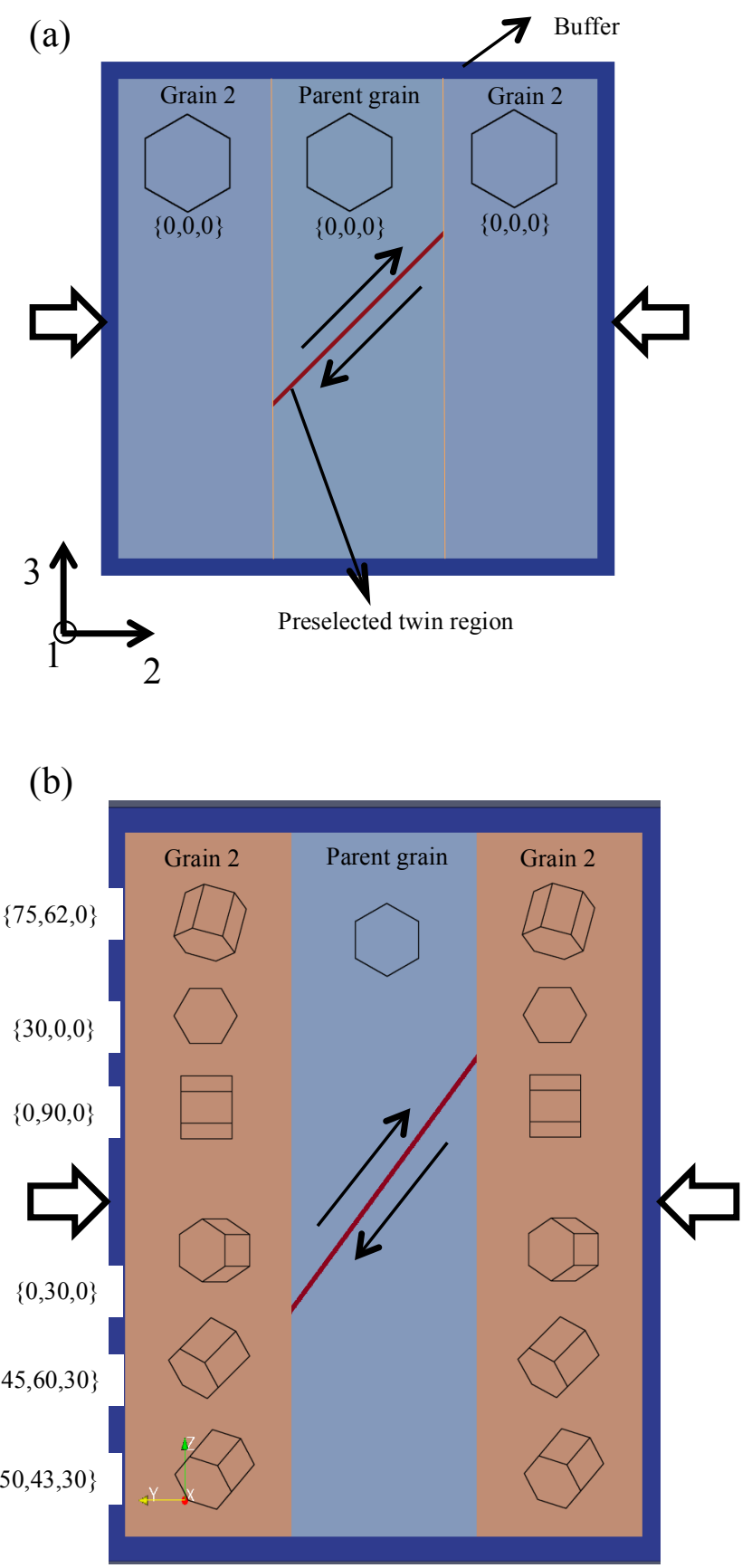

Figure 2: Schematic of the tri-crystal unit cell used in the simulations. (a) Unit cell representing a twin inside a single crystal grain; (b) Unit cell representing a twin front arrested at the grain boundary. Wireframe of the neighbor orientations used in the simulations are shown, together with their associated Euler angles, as seen looking along the z-axis. 
(a)

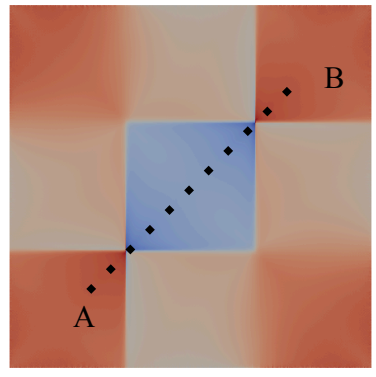

(b)

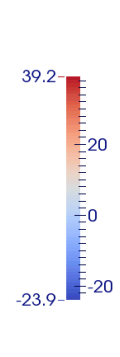

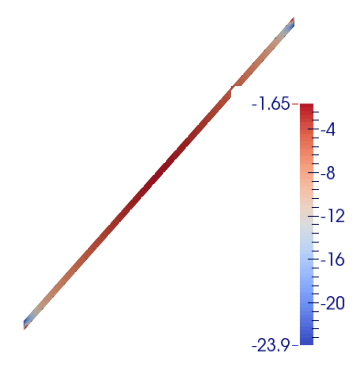

(c)

(d)

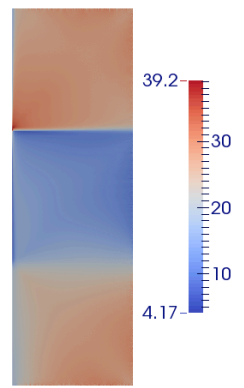

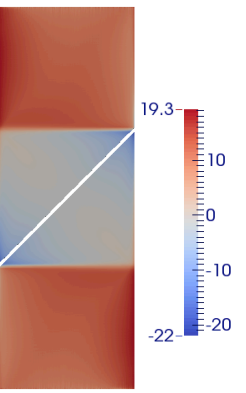

Figure 3: Distribution of the twin-resolved shear stress (T-RSS) at the end of the transformation process for Case 0, under an applied stress of $-40 \mathrm{MPa}$. (a) Whole unit cell (max: 39.2, min=$23.9 \mathrm{MPa})$; (b) twin domain $(\max =-1.65, \min =-23.9 \mathrm{MPa}$, (c) neighbor grain $(\max =39.2$, $\min =4.2 \mathrm{MPa})$; (d) parent grain $(\max =19.3, \min =-22.0 \mathrm{MPa})$. 


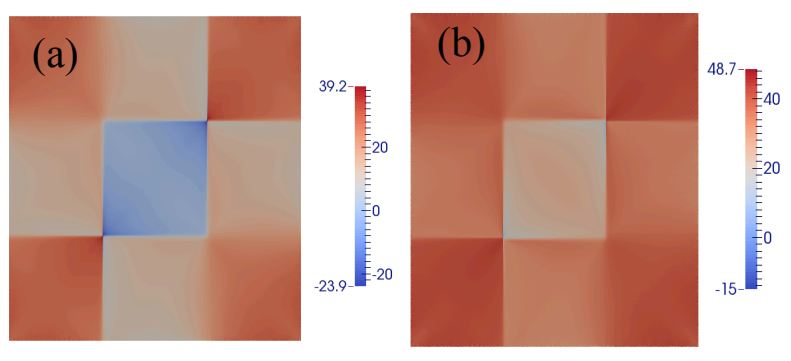

(c) Case 0

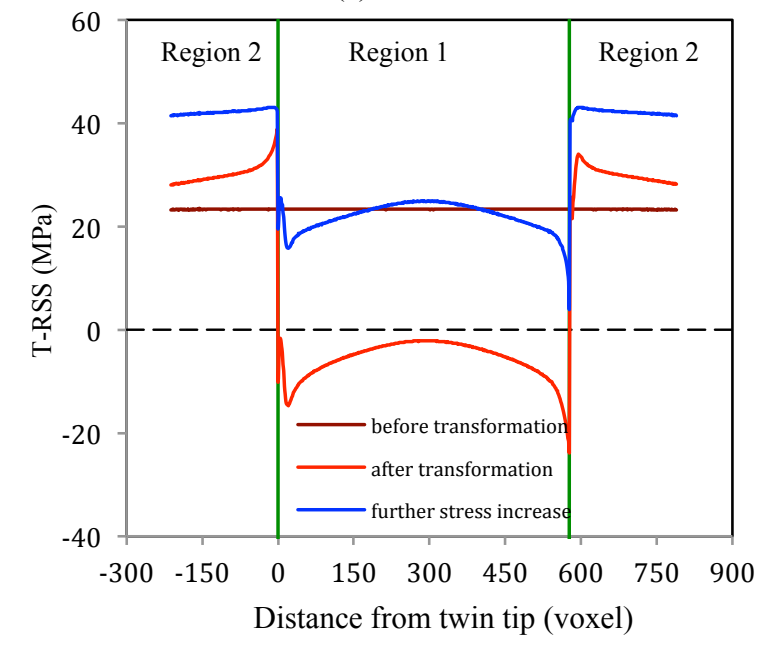

(d) Case 1

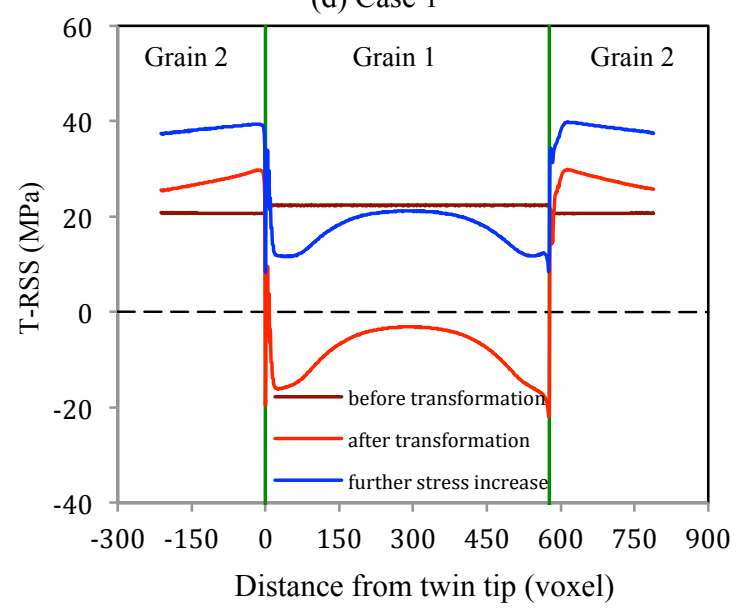

Figure 4: Full cell contour plots for Case 0: (a) at the end of transformation process; (b) upon subsequent increase of applied stress to $-60 \mathrm{MPa}$. Twin shear stress (T-RSS) profiles for (c) Case 0 and (d) Case 1 along the top facet of the twin, from point $A$ to point $B$. The three profiles correspond to: before the twin transformation process under applied $-40 \mathrm{MPa}$; at the end of transformation; and upon subsequent increase of stress to -60 MPa. 


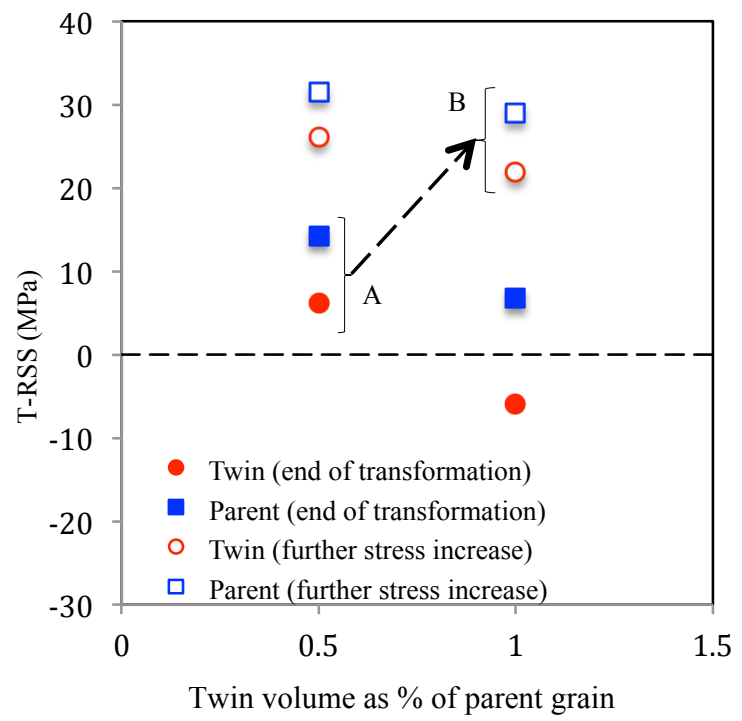

Figure 5: Effect of the twin volume fraction on the average T-RSS values in the parent and the twin. Results correspond to Case 0. Solid symbols: values at the end of the transformation process $(-40 \mathrm{MPa})$. Open symbols: values after increasing the applied stress to $-60 \mathrm{MPa}$ 

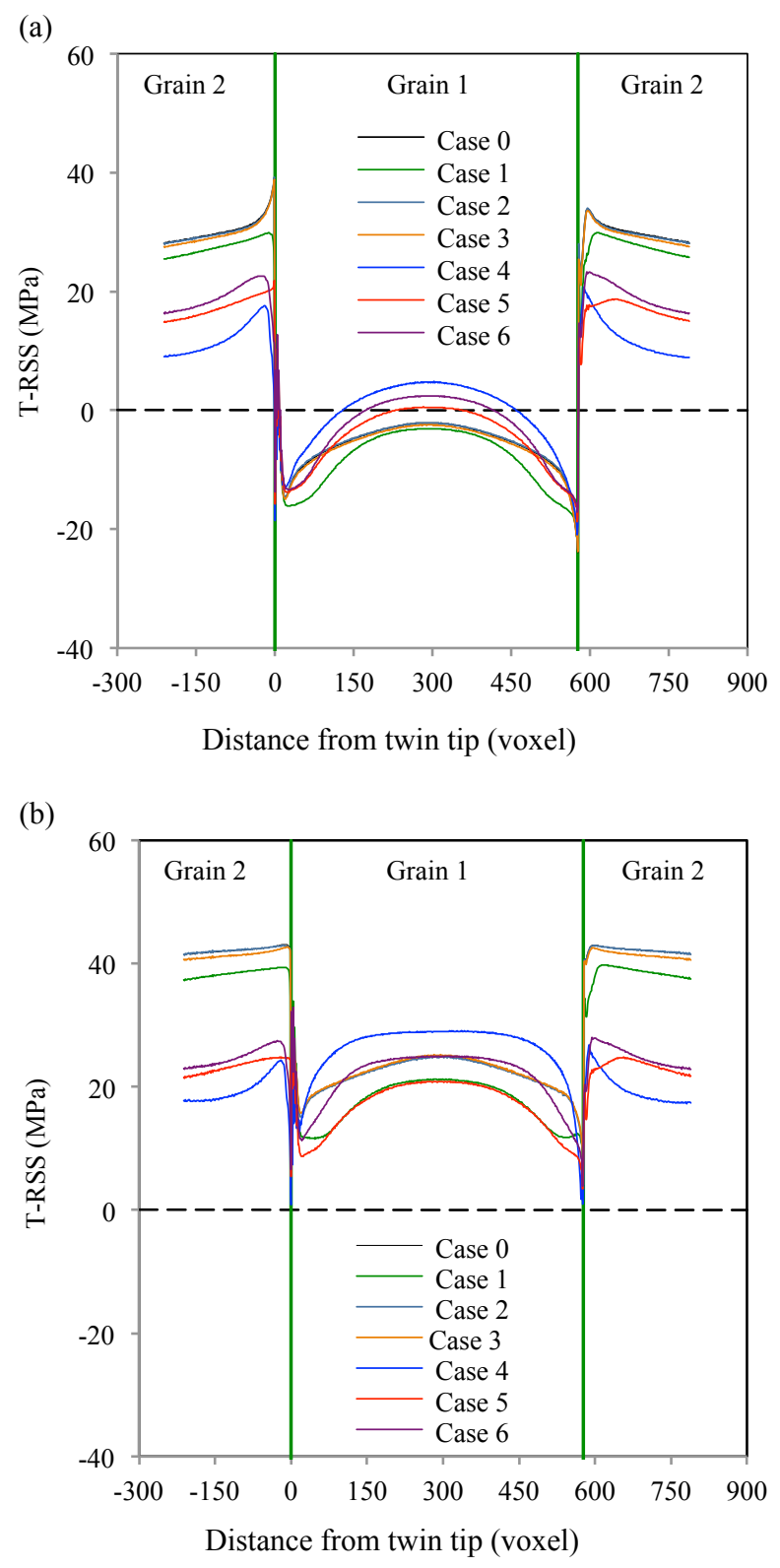

Figure 6: Twin shear stress (T-RSS) profiles at the twin-matrix interface in the parent grain for the 7 different neighbor orientations considered here: (a) at end of the transformation process (b) upon further increase in the stress $(-60 \mathrm{MPa})$. The values correspond to voxels along the upper twin interface. 